\title{
Vibration Analysis of Simply Supported Rectangular Tank Partially Filled with Water
}

\author{
Kamila Kotrasová ${ }^{1, *}$ \\ ${ }^{1}$ DSM, ISE, Civil Engineering Faculty, Technical University of Košice, 04200 Košice, Slovakia
}

\begin{abstract}
Ground-supported tanks are used as fluid storage. One of the phenomena associated with the seismic response of liquid-filled tanks is the fluid motion occurring that causes "sloshing" at the top of free surface. This paper presents the theoretical of fluid response of rectangular tank due to horizontal acceleration of tank bottom, the impulsive and convective (sloshing) pressure and the fluid natural frequencies. The vibration analysis of fluid filled rectangular container was monitored and was evaluated in experiment for purpose to evaluation of the first frequency mode and vibration response of fluid were analysed by using FEM
\end{abstract}

\section{Introduction}

The natural vibration analysis of solids in contact with fluid has been an issue for many years, and there are many researchers who have dealt with this challenging problem.

It is knows that fluid in partially filled containers tends to experience under certain conditions. When frequency of the container motion is close to the natural frequency of fluid in the container, localized hight impact load on the tank walls occur due to extreme liquid motion [1]. Mane researchers have investigated sloshing phenomena using various analytic, numerical, and experimental approached, and these syudies have revealed many significant phenomena in recent decades [2-4].

The potential formulation is often used in sloshing studies. This approach provides a simplified means of evaluating sloshing, but its use is limited in the case of real nonlinear liquid problems [5-7]. Many potential flow theory is not sufficiently reliable in many numerical studies based on Navier-Stokes equations have been performed to overcome the aforementioned problem. The calculation of the free surface profile inside a container is a key factor in the accurate approximation of loads generated by sloshing. As a result, many studies have been conducted using Two-Dimensional (2-D) and ThreeDimensional (3-D) simulations with the use of freesurface capturing models such as the Marker and Cell (MAC) approach, the Volume of Fluid method (VOF), and the Level Set Method (LSM), or a combination of LSM and VOF [8-10]. Also, recently, the Smoothed Particle Hydrodynamics (SPH) approach has been quite successful in simulating 2-D sloshing flows.

Using numerical tools, investigators have examined characteristics of sloshing phenomena that depend on design variables such as depth of fluid, geometry of container, the frequency and amplitude of external excitation, and the position of the center of gravity. To suppress extreme sloshing, it is important to carefully consider these design variables. One of the solutions used to prevent extreme free surface fluctuations is to install baffles inside a liquid tank [11]. Many researchers have successfully demonstrated that installing baffles is useful in reducing the pressure loads on the walls or ceiling of a tank in combination with suppressing extreme fluid motion, using numerical simulation based on the NS equations. The filling level in the tanks is also a key element. The largest sloshing loads tend to occur at filling level $(H)$ and tank length $(L)$ filling ratios of $0.1 \leq H / L \leq$ $0.5[12]$.

Most of the aforementioned studies focused on a frequency of external excitation that is close to the natural frequency. However, to our knowledge, it is hard to find reports that describe a wide frequency ratio range for sloshing in 2-D and 3-D tanks, and especially for viscous flow conditions. Recently [12,13] focused on sway motion (horizontal amplitude excitation) and considered a broad range of frequencies, filling conditions, and amplitude excitations. However, the filling ratios they examined are quite small (to satisfy the shallow water regimes). A narrow tank has been used to limit 3-D effects and allow for an extensive study of 2-D waves.

The phenomenon of motion of liquids when fluid in containers can be excited and has an unrestrained surface, was referred as "sloshing". Liquid sloshing phenomena can be triggered e.g. by seismic effects in stationary containers or can be initiated by movements of the container itself. The sloshing characteristically of is a

\footnotetext{
* Corresponding author: kamila.kotrasova@tuke.sk
} 
wave motion from side to side within the container [14$16]$.

The motion of the liquid storage tanks is, due to the high complexity of the problem, in fact, really complicated task. Number of particular problems should be taken into account, for example:

- dynamic interaction between contained fluid and tank,

- sloshing motion of the contained fluid,

- dynamic interaction between tank and sub-soil [17].

The fluid flows causes sloshing of free surface. The free liquid surface may experience different types of motion including simple planar, non-planar, rotational, irregular beating, symmetric, asymmetric, quasi-periodic and chaotic $[18,19]$. The type of motion is depended on the container shape and type of excitation. The amplitude of slosh depends on the liquid-fill depth, liquid properties, tank geometry, frequency and amplitude of the tank motion. The fluid resonance in the case of horizontal excitation is activate when the external exciting frequency is close to the natural frequency of the liquid [20-24].

The liquid sloshing is important task with regard to the reliability and safety structures, because an eventual damages of containers used for storage of hazardous liquids, e.g. petroleum, chemical and radioactive waste, are catastrophic, consequences are financial, and environmental loses [25-27]. The knowledge of sloshing phenomenon of the contained fluid, dynamic interaction between contained fluid and tank, hydrodynamic pressures, as well as frequency properties of tank-fluid systems are played fundamental role for a reliability design of earthquake-resistant structures/facilities - tanks [28-31].

\section{Contents of paper}

1 Introduction

2 Analysis of tank - fluid system

2.1 The hydrodynamic pressure

2.1.1 The hydrodynamic impulsive pressure

3 FEM numerical simulation

3.1 The incompressible Navier-Stokes equations

4 Analysis, results and conclusion

Acknowledgment

References

\section{Analysis of tank-fluid system}

The rectangular tank with rigid walls exited by horizontal excitation is considered. The hydrodynamic pressure may be obtained from the solution of Laplace's equation. The total hydrodynamic pressure is given as the sum of impulsive and convective pressure contribution [32]

$$
p=p_{i}+p_{c} \text {. }
$$

A rectangular container with rigid walls having horizontal acceleration $\ddot{u}_{o}$ in the $x$-direction (see Figure 1a) is considered [33]. Due to the acceleration $\ddot{u}_{o}$, the hydrodynamic pressures on the tank walls and tank bottom are generated The tank has depth $H$, length $2 L$, and unit wall thickness [34,35].

\subsection{The hydrodynamic pressure}

\subsubsection{The hydrodynamic impulsive pressure}

The horizontal component of the fluid velocity $\dot{u}$ is independent of the $y$-coordinate, therefore the behaviour of the fluid may be simulated as thin, massless, vertical membranes that are free to move in the $x$-direction. The distance between neighbouring membranes is $d x$ (Figure 1b) [36].

If the walls of the tank are excited by acceleration, the membranes do accelerate proportional to the fluid while, whrereas the same time, the fluid squeezes vertically along membranes vertically along $y$-direction.

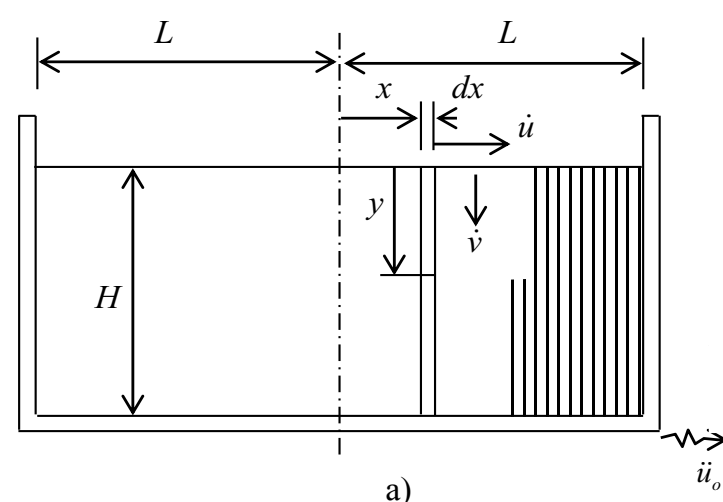

a)

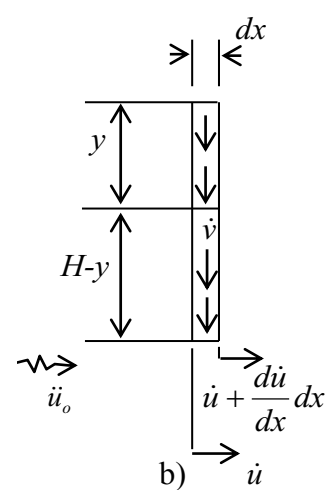

Fig. 1. a) Rectangular tank-fluid system, b) vertical fluid element.

The vertical velocity $\dot{v}$ of fluid between two adjacent membranes is dependent on the horizontal velocity $\dot{u}$, Figure $1 \mathrm{~b}$, according to eq. (2)

$$
\dot{v}=(H-y) \frac{d \dot{u}}{d x} .
$$

Due to incompressibility of the fluid, the acceleration $\ddot{v}$ is proportional to the velocity $\dot{v}$ and the acceleration $u$ is proportional to the velocity $\dot{u}$, and the pressure in the 
fluid between two membranes is given by the standard hydrodynamic eq. (3)

$$
\frac{\partial p}{\partial y}=-\rho \ddot{v},
$$

where $\rho$ is density of the fluid.

Total horizontal force on one membrane is

$$
F=\int_{o}^{H} p d y .
$$

therefore

$$
\begin{aligned}
p & =-\rho \int_{0}^{y}(H-y) \frac{d \ddot{u}}{d x} d y= \\
& =-\rho H^{2}\left(\frac{y}{H}-\frac{1}{2}\left(\frac{y}{H}\right)^{2}\right) \frac{d \ddot{u}}{d x}
\end{aligned}
$$

and

$$
\begin{aligned}
F & =-\rho H^{2} \int_{0}^{H}\left(\frac{y}{H}-\frac{1}{2}\left(\frac{y}{H}\right)^{2}\right) \frac{d \ddot{u}}{d x} d y= \\
& =-\rho \frac{H^{3}}{3} \frac{d \ddot{u}}{d x}
\end{aligned}
$$

The kinetic energy of the fluid is

$$
W_{k}=\int_{-L 0}^{+L H} \int_{0} \frac{1}{2} \rho\left(\dot{u}^{2}+\dot{v}^{2}\right) d x d y .
$$

The potential energy of the fluid is zero, consequently Hamilton's principle states that

$$
\delta \int_{t_{1}}^{t_{2}}\left(W_{k}-W_{p}\right) d t=0 .
$$

The equation of motion is

$$
\frac{d^{2} \dot{u}}{d x^{2}}-\frac{3}{H^{2}} \dot{u}=0 .
$$

The acceleration $\ddot{u}_{0}$ produces an increase in the pressure on one wall and a decrease in the pressure on the other wall

$$
p_{i w}=\rho \ddot{u}_{0} H\left(\frac{y}{H}-\frac{1}{2}\left(\frac{y}{H}\right)^{2}\right) \sqrt{3} \tanh \sqrt{3} \frac{L}{H} .
$$

The dimensionless distance is given $\xi=z / H$, where $z=H-y$, then

$$
\begin{aligned}
& p_{i w}\left(\frac{z}{H}\right)= \\
& =\rho \ddot{u}_{0} H \frac{\sqrt{3}}{2}\left(1-\left(\frac{z}{H}\right)^{2}\right) \tanh \sqrt{3} \frac{L}{H}
\end{aligned}
$$

Hydrodynamic impulsive pressure on the wall of tank are as well given by

$$
p_{i w}(\xi)=C_{i w}(\xi) \rho H A_{g}(t),
$$

where $A_{g}(t)$ represents the free-field motion of the ground, and $\rho$ is mass density of the liquid $[37,38]$.

The function $C_{i w}(\xi)$ gives the distribution of hydrodynamic pressures $p_{i w}(\xi)$ along the height of wall

$$
C_{i w}(\xi)=\frac{\sqrt{3}}{2}\left(1-\xi^{2}\right) \tanh \sqrt{3} \frac{L}{H} .
$$

\subsubsection{The hydrodynamic convective pressure}

The effect of impulsive pressures causes the oscillation of the fluid. The fluid constrained between the rigid membranes having free to rotate, Figure 2, is given

$$
\begin{gathered}
\dot{u}=\frac{L^{2}-x^{2}}{2} \frac{d \dot{\theta}}{d y}, \\
\dot{v}=\dot{\theta} z
\end{gathered}
$$

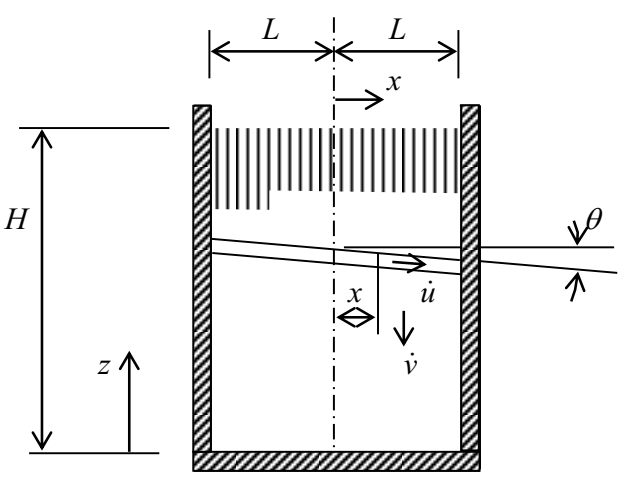

Fig. 2. Rectangular fluid filled tank.

The pressure of the fluid is given

$$
\frac{\partial p}{\partial x}=-\rho \ddot{u} .
$$

The hydrodynamic convective pressures are given

$$
p_{c w}=\left(\rho \frac{L^{3}}{3} \sqrt{\frac{5}{2}} \frac{\cosh \sqrt{\frac{5}{2}} \frac{x}{L}}{\sinh \sqrt{\frac{5}{2}} \frac{H}{L}}\right) \omega^{2} \theta_{0} \sin \omega t .
$$

In dependence on $\xi=z / H$, the convective hydrodynamic pressure is given by a summation of modal terms (sloshing modes), each one having a different variation with time

$$
p_{H D w n}(\xi)=\sum_{n=1}^{\infty} Q_{c w n}(\xi) \rho L A_{n}\left(f_{c n}\right) .
$$

The dominant contribution for a rectangular container is the fundamental mode

$$
p_{H D c w 1}(\xi)=Q_{c w 1}(\xi) \rho L A_{1}\left(f_{c 1}\right),
$$

Where the free-field motion of the ground is represented by the peak value of $A_{1}(t)$, it is the acceleration response function of a simple oscillator having the frequency of the first mode, the appropriate value of the damping of an input acceleration $A_{g}(t) . \rho$ is mass density of the liquid. The function $Q_{c w 1}(\xi)$ defines the distribution of hydrodynamic pressures $p_{c w 1}(\xi)$ along the height of wall 


$$
Q_{c w 1}(\xi)=0,833 \frac{\cosh \left(\xi \frac{1}{2} \sqrt{\frac{5}{2}} \frac{H}{L}\right)}{\cosh \left(\frac{1}{2} \sqrt{\frac{5}{2}} \frac{H}{L}\right)} .
$$

The equation of the motion of a slice of the fluid is

$$
\int_{-l}^{+l} \frac{\partial p}{\partial y} d y x d x=-\rho \frac{\left(2 L^{3}\right)}{12} \ddot{\theta} d y .
$$

The solution of eq. (20) respecting the boundary conditions appropriate to the problem, is for sinusoidal oscillations

$$
\theta=\theta_{0} \frac{\sinh \sqrt{\frac{5}{2}} \frac{y}{L}}{\sinh \sqrt{\frac{5}{2}} \frac{H}{L}} \sin \omega t .
$$

This specifies the oscillation of the fluid. To determine the natural frequency of vibration, the maximum kinetic energy, $W_{K}$, is equated to the maximum potential energy, $W_{P}$ :

$$
\begin{gathered}
W_{K}=\int_{0}^{h+l} \int_{-l}^{1} \frac{1}{2} \rho\left(u^{2}+v^{2}\right) \omega^{2} \sin ^{2} \omega t d x d y, \\
W_{P}=\int_{-l}^{+l} \frac{1}{2} \rho g x^{2} \sin \omega t d x .
\end{gathered}
$$

That gives

$$
\omega^{2}=\frac{g}{L} \sqrt{\frac{5}{2}} \tanh \sqrt{\frac{5}{2}} \frac{H}{L} .
$$

The circular frequency for the $n^{\text {th }}$ mode is

$$
\omega_{n}^{2}=\frac{g}{L} n \sqrt{\frac{5}{2}} \tanh n \sqrt{\frac{5}{2}} \frac{H}{L} .
$$

\section{FEM numerical simulation}

The finite element method (FEM) is well established for the simulation of complex engineering problems involving structures and fluids [39].

\subsection{The incompressible Navier-Stokes equations}

We consider a fluid, liquid, or gas, moving in a domain $\Omega$. The fluid motion is a difficult task since this is a nonlinear physical phenomenon involving many unknowns. The main unknowns are the mass density, the pressure, the velocity, and the temperature, but the list may be longer depending on the particular case.

The incompressible Navier-Stokes equations for Newtonian fluids is given by

$$
\begin{gathered}
\partial_{t} \mathbf{v}+(\mathbf{v} \cdot \nabla) \mathbf{v}-\nabla \cdot(2 v D \mathbf{v})+\nabla p=\mathbf{f}, \\
\nabla \cdot \mathbf{v}=0,
\end{gathered}
$$

where $\mathbf{v}$ is the velocity of the flow, $p$ is pressure, and its deformation tensor is $D \mathbf{v}=(1 / 2)\left(\nabla \mathbf{v}+\nabla \mathbf{v}^{t}\right)$. The momentum equation (28) is inherited from Newton's law, while equation (29) is the mass conservation equation for incompressible flows.

The general mass conservation equation satisfied by $\mathbf{v}$ and $\rho$ is given by

$$
\partial_{t} \rho+\nabla \cdot(\mathbf{v} \rho)=0
$$

The pressure and velocity had to define for the fluid domain and the additional special conditions are considered:

- free surface, the interface between fluid and gas;

- common boundary between solid and fluid.

\section{Analysis, results and conclusion}

The rectangular tank made of glass filled with water was used in the experiment. The tank's inner bottom dimensions were $19.2 \mathrm{~cm}$ and $39.2 \mathrm{~cm}$. The inner tank height was $24.2 \mathrm{~cm}$, consequently it is dimension of full fluid filling.

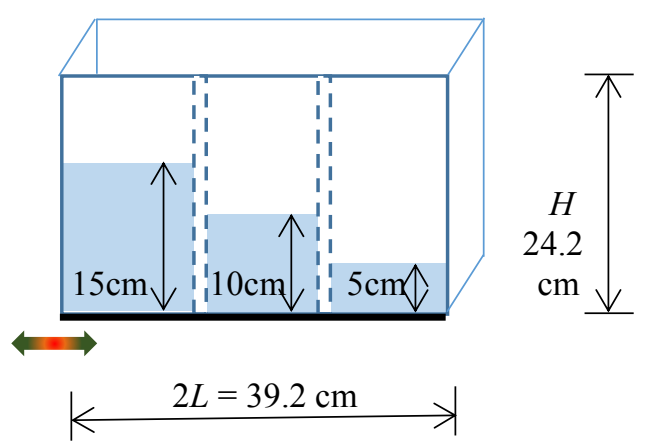

Fig. 3. The model of rectangular tank with variable fluid filling $5 \mathrm{~cm}, 10 \mathrm{~cm}$ and $15 \mathrm{~cm}$.

The comparing of the first eight circular frequencies for tank fluid filling using eq. (26) was seen in Figure 4. The values of first seven circular frequencies for tank fluid filling using eq. (26) are different. The eight and next natural frequencies for the fluid filling of height $5 \mathrm{~cm}, 10$ $\mathrm{cm}$ and $15 \mathrm{~cm}$ give the same values.

A glass rectangular tank was used in the experiment. The tank was excited by a horizontal harmonious motion with various frequencies with amplitude $0.5 \mathrm{~cm}$ in the direction of tank longer bottom side $39.2 \mathrm{~cm}$.

The height of water filling in the container was $5 \mathrm{~m}$, $10 \mathrm{~cm}$ and $15 \mathrm{~cm}$. The tank was excited by a horizontal harmonious motion of various frequencies with amplitudes of $0.5 \mathrm{~cm}$ in the direction of tank longer bottom side $39.2 \mathrm{~cm}$, see Figure 3 . 


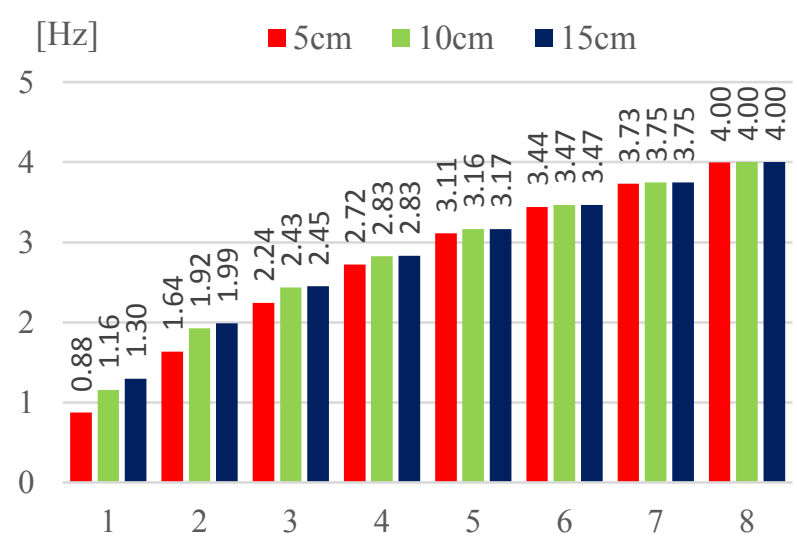

Fig. 4. The comparison of first five fluid natural frequencies for $5 \mathrm{~cm}, 10 \mathrm{~cm}$ and $15 \mathrm{~cm}$ fluid filling.

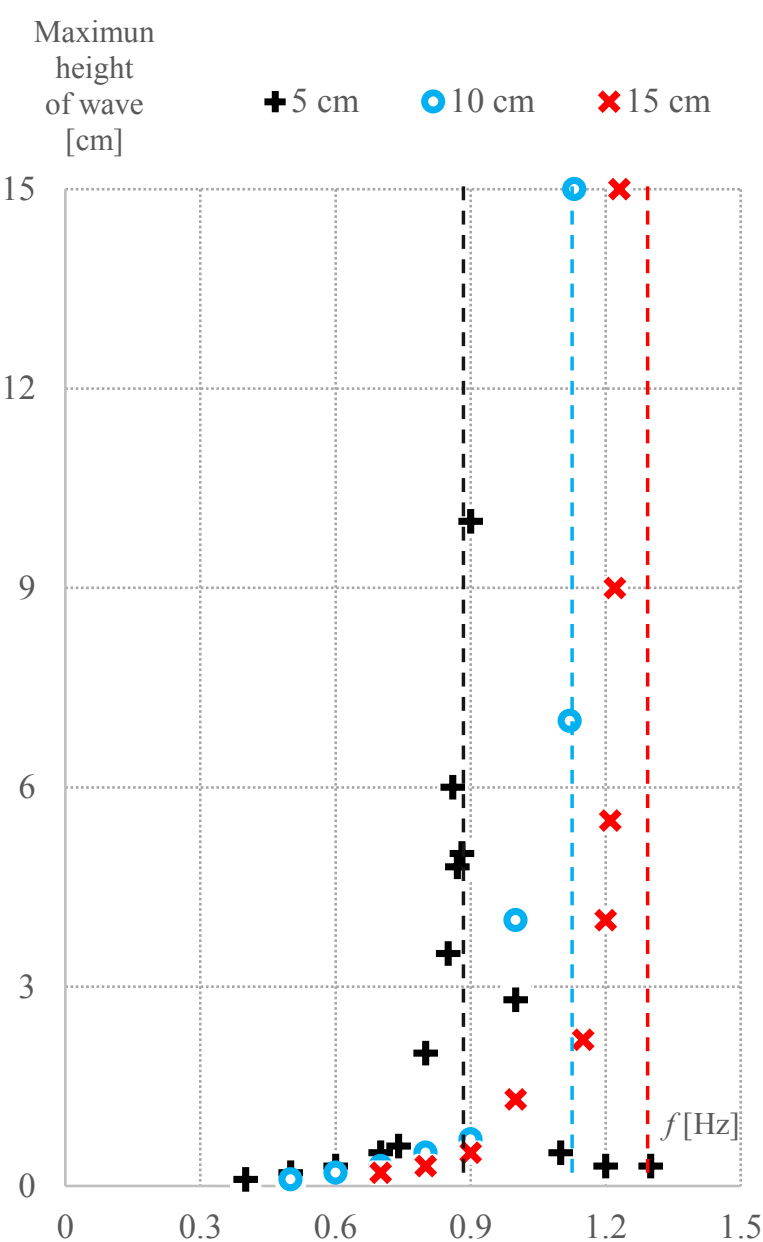

Fig. 5. The peak values of waves for 5,10 and $15 \mathrm{~cm}$ fluid filling of the tank.

The peak waves of fluid for $5 \mathrm{~cm}$ (black “+”), $10 \mathrm{~cm}$ (blue "o"), $15 \mathrm{~cm}$ (red "x") of fluid filling was shown in Figure 5 for different frequencies with amplitude $0.5 \mathrm{~cm}$. Using eq. (26), the first frequencies of the glass rectangular fluid filled tank were calculated.

The peak wave of fluid for $5 \mathrm{~cm}$ fluid filling (black "+" in Figure 5) in tank was $10 \mathrm{~cm}$ from the original free surface, excited frequency was $0.9 \mathrm{~Hz}$. The first circular frequency was expected as $0.875 \mathrm{~Hz}$, showed by vertical black dash line in Figure 5.

The peak wave of fluid for $10 \mathrm{~cm}$ fluid filling (blue "o" in Figure 5) in tank was fluid splashing when the tank was excited with frequency is $1.13 \mathrm{~Hz}$. The first circular frequency is $1.156 \mathrm{~Hz}$, showed by vertical blue dash line in Figure 5.

The peak wave of fluid for $15 \mathrm{~cm}$ fluid filling (red " $\mathbf{x}$ " in Figure 5) in tank was fluid splashing when the tank was excited with frequency is $1.23 \mathrm{~Hz}$. The first circular frequency was expected as $1.29 \mathrm{~Hz}$, showed by vertical red dash line in Figure 5.

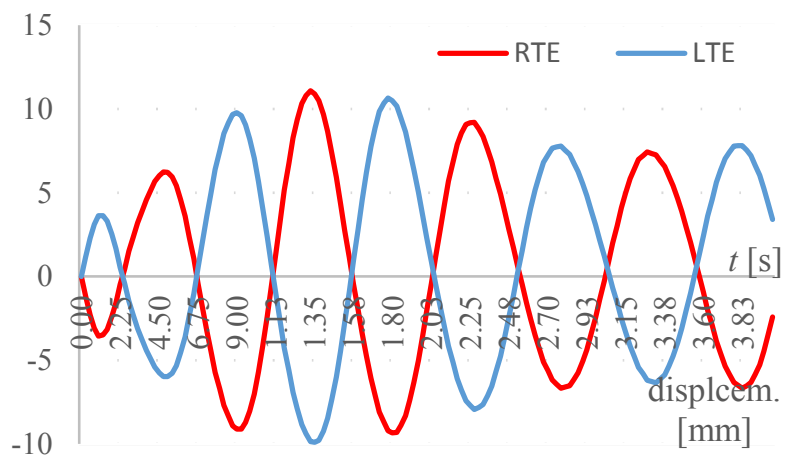

Fig. 7. Time-depended response of fluid vertical displacement on the free surface in node RTE and LTE.

The Figure 6 shows the time-depended response of fluid vertical displacement in fluid domain node LTE (left top edge) and RTE (left top edge)).

\section{Acknowledgment}

This paper has been supported by the project VEGA 1/0477/15 and VEGA 1/0374/19.

\section{References}

1. R.L. Bass, E.B. Bowles, P.A. Cox, P.A., Society of Naval Architects and Marine Engineers, 88, pp.103126.

2. H. Akyildiz, E. Unal, Ocean Engineering, 32(11-12), pp.1503-1516.

3. H. Akyildiz, N.E. Unal, Ocean Engineering, 33(16), pp.2135-2149.

4. Y. Kim, Int. J. Nav. Archit. Ocean Eng., 5(2), pp.227245.

5. Y. Kim, Y. Shin, Y. K.H. Lee, Applied Ocean Research, 26(5), pp.213-226.

6. C.S. Lee, J.R. Cho, W.S. Kim, B.J. Noh, M.H. Kim and J.M. Lee, Int. J. Nav. Archit. Ocean Eng., 5(1), pp.1-20 (2013).

7. G.J. Lee, Int. J. Nav. Archit. Ocean Eng., 6(1), pp.132-150 (2014). 
8. V. Armenio, M.L. Rocca, Ocean Engineering, 23(8), pp.705-739.

9. W.G. Price, Y.G. Chen, International Journal for Numerical Methods in Fluids, 51(3), pp.305-330.

10. S.H. Rhee, S.H., Journal of Fluids Engineering Ameriacan Society of Mechanical Engineers, 127(3), pp.572-582.

11. R.A. Ibrahim, Liquid sloshing dynamics: theory and applications. New York: Cambridge University Press (2005)

12. J. MacDonald, J. Maguire, 23rd Gastech conference, Bangkok (2015).

13. G.S. Brar, S. Singh, Procedia Technology, 14(2014):490-496.

14. B. Bouscasse, M. Antuono, A. Colagrossi, C. Lugni, International Journal of Nonlinear Sciences and Numerical Simulation, 14(2), pp.123-138.

15. J.H. Jung, H.S. Yoon, Ch.Y. Lee, Int. J. Archit. Ocean Eng. 7(2015):580-594.

16. H. N. Abramson, The dynamic behavior of liquids in moving containers. NASA SP-106, National Aeronautics and Space Administration, Washington, D. C., 1966.

17. P. Pal, International Journal of Recent Trends in Engineering, 1(2009)8:1-5.

18. J. Protivinsky, M. Kubzova, M. Krejsa, Key Engineering Materials, 738(2017):195-204.

19. V. Michalcova et al., Applied Mechanics and Materials, 617(2014):275-279.

20. B. Taraba, Z Michalec, V. Michalcová, T. Blejchar, M Bojko, M Kozubkova, Fuel. 118(2014):107-112.

21. M. Žmindak, I. Grajciar, Computers and Structures. 64(1997):(5-6)(1155-1164.

22. M. Krejsa. P. Janas, V. Krejsa, International Journal of Mathematics and Computers in Simulation. 8(2014):121-126.

23. K. Kotrasova, Advanced Materials Research. 969(2014):320-323.

24. V. Michalcova, L. Lausová, Computers and Structures. (2017), in press.

25. A. Bouabidi, Z. Driss, N. Cherif, M. S. Abid, WSEAS Transactions on Fluid Mechanics. 11(2016):1-9.

26. K. Kotrasova, Procedia Engineering. 190(2017):2-6.

27. M. Mihalikova, M. Hagarova, D. Jakubeczyova, J. Cervova, A. Liskova, International Journal of Electrochemical Science, 11(2016): (6)4206-4218.

28. O. Sucharda, J. Brozovsky, International journal of mechanics, 7(2013):(3)192-200.

29. K. Tvrda, Procedia Engineering. 190(2017):516521.

30. G. Lajcakova, J. Melcer, Comunications : Scientific Letters of the University of Žilina. 13(2011):(3)1418.

31. M. Major, K. Kuliński, I. Major, Procedia Engineering. 190(2017):231-236.
32. E. Andriyanova, V. Astafev, WSEAS Transactions on Fluid Mechanics. 11(2016):10-17.

33. M. Ramesh, P. Kuklik, M. Válek, Procedia Engineering. 195(2017):73-80.

34. K. Kotrasova, Boundary Field Problems and Computer Simulation, 54(2015):17-22.

35. M. H. Hsieh, et al. Journal of Vibration Engineering and Technologies, 5(2017):(5)423-428.

36. J. Králik, Advanced Materials Research. 688(2013):213-221.

37. G. W. Housner, "Earthquake pressures on fluid containers," California institute of technology, Pasadena California, 1954.

38. EN 1998-4: 2006 Eurocode 8. Design of structures for earthquake resistance. Part 4: Silos, tanks and pipelines. CEN, Brussels, 2006.

39. K. Kotrasova, I. Grajciar, Advanced Materials Research, 969(2014):119-124. 